\title{
ON STRICT AND SIMPLE TYPE EXTENSIONS
}

\section{MOHAN TIKOO}

Department of Mathematics

Southeast Missouri State University

Cape Girardeau, Missouri 63701 U.S.A.

(Received August 14, 1996 and in revised form November 21, 1996)

\begin{abstract}
Let $(Y, \tau)$ be an extension of a space $\left(X, \tau^{\prime}\right) . p \in Y$, let $0_{y}^{p}=\{W \cap X: W \in \tau, p \in W\}$. For $U \in \tau^{\prime}$, let $o(U)=\left\{p \in Y: U \in O_{y}^{p}\right\}$. In 1964, Banaschweski introduced the strict extension $Y^{\#}$, and the simple extension $Y^{+}$of $X$ (induced by $(Y, \tau)$ ) having base $\left\{o(U): U \in \tau^{\prime}\right\}$ and $\left\{U \cup\{p\}: p \in Y\right.$, and $\left.U \in O_{y}^{P}\right\}$, respectively. The extensions $Y^{\#}$ and $Y^{+}$have been extensively used since then. In this paper, the open filters $\mathcal{L}^{p}=\left\{W \in \tau^{\prime}: W \supseteq \operatorname{int}_{X} \mathrm{cl}_{X}(U)\right.$ for some $\left.U \in O_{y}^{p}\right\}$, and $\mathcal{U}^{p}=\left\{W \in \tau^{\prime}:\right.$ int $\left._{X} \mathrm{cl}_{X}(W) \in O_{y}^{p}\right\}=\left\{W \in \tau^{\prime}:\right.$ int $\left._{x} \mathrm{cl}_{X}(W) \in \mathcal{L}^{p}\right\}=\cap\{U: U$ is an open ultrafilter on $\left.X, O_{X}^{p} \subset \mathcal{U}\right\}$ on $X$ are used to define some new topologies on $Y$. Some of these topologies produce nice extensions of $\left(X, \tau^{\prime}\right)$. We study some interrelationships of these extensions with $Y^{\#}$, and $\mathrm{Y}^{+}$ respectively.
\end{abstract}

KEY WORDS AND PHRASES: Extension, simple extension, strict extension, H-closed, s-closed, almost realcompact, near compact.

1980 AMS SUBJECT CLASSIFICATION CODES: Primary 54D25.

\section{INTRODUCTION}

A topological space $Y$ is an extension of a space $X$ if $X$ is a dense subspace of $Y$. If $Y_{l}$ and $Y_{2}$ are two extensions of a space $X$, then $Y_{2}$ is said to be projectively larger than $Y_{1}$, written $Y_{2} \geq Y_{1}$ (or $Y_{1} \leq Y_{2}$ ), provided that there exists a continuous map $f: Y_{2} \rightarrow Y_{1}$ such that $\left.f\right|_{X}=i_{X}$, the identity map on $X$. Two extensions $Y_{1}$ and $Y_{2}$ of $X$ are called equivalent if $Y_{1} \leq Y_{2}$ and $Y_{2} \leq Y_{1}$. We shall identify two equivalent extensions of $X$. With this convention, the class $E(X)$ of all the Hausdorff extensions of a Hausdorff space $X$ is a set. Let $(Y, \tau) \in E(X)$ and let $p \in Y$. If $N_{p}$ is the open neighborhood filter of $p$ in $Y$, the set $O_{Y}^{p}=\left\{N \cap X: N \in N_{p}\right\}$ (called the trace of $N_{p}$ on $X$ ) is an open filter on $X$. If $U$ is open in $X$, denote

$$
o_{Y}(U)=\left\{p \in Y: U \in O_{Y}^{p}\right\}
$$


In 1964 Banaschewski [1] introduced the extensions $\mathrm{Y}^{\#}$ (resp. $\mathrm{Y}^{+}$) the strict extension (resp. the simple extension) of $X$ induced by $Y$ satisfying $Y^{*} \leq Y \leq Y^{+}$. The topology $\tau^{*}$ on $Y^{*}$ (resp. $\tau^{+}$on $Y^{+}$) has for an open base the collection $\left\{o_{y}(U): U\right.$ open in $\left.X\right\}$ (resp., the collection $\{U \cup\{p\}: p \in Y$, and $\left.U \in O_{Y}^{p}\right\}$ ). The extensions $Y^{\#}$, and $Y^{+}$have been studied extensively and have proved extremely useful regarding some properties weaker than compactness, such as nearly compact, almost realcompact, feebly compact, $H$-closed, $s$-closed, etc.. In this paper we introduce new extensions $Y^{l}, Y^{\mu}, Y^{Y^{*}}$, and $Y^{\mu^{*}}$, study some of their properties, and compare them with $Y, Y^{\#}$, and $Y^{+}$. All spaces under consideration are Hausdorff.

\section{THE EXTENSIONS $Y^{l}$ AND $\boldsymbol{Y}^{\mu}$.}

In this section, we introduce several topologies on $Y$, and compare them with $\tau$. Some of these topologies yield interesting extensions of $\left(X, \tau^{\prime}\right)$.

DEFINITION 2.1. Let $(Y, \tau)$ be an extension of a space $\left(X, \tau^{\prime}\right)$. For $p \in Y$ define

$$
\begin{gathered}
\mathcal{U}^{p}=\left\{W: W \in \tau^{\prime}, \text { int }_{X} \mathrm{cl}_{X} W \in O_{Y}^{p}\right\}, \\
\mathcal{L}^{p}=\left\{W: W \in \tau^{\prime}, W \supseteq \operatorname{int}_{X} c l_{X} U \text { for some } U \in O_{Y}^{p}\right\} .
\end{gathered}
$$

\section{LEMMA 2.1.}

(a) Both $\mathcal{U}^{p}$ and $\mathcal{L}^{p}$ are open filters on $X$ such that $\mathcal{L}^{p} \subseteq O_{Y}^{p} \subseteq \mathcal{U}^{p}$.

(b) $\mathcal{U}^{p}=\left\{W: W \in \tau^{\prime}\right.$, int $\left._{X} \mathrm{cl}_{X} W \in \mathcal{L}^{p}\right\}$

$$
=\cap\left\{U: U \text { is an open ultrafilter on } X, O_{Y}^{p} \subset \mathcal{U}\right\}
$$

PROOF. We prove (b). Let $W=\left\{W: W \in \tau^{\prime}\right.$, int $\left._{x} \mathrm{cl}_{x} W \in \mathcal{L}^{p}\right\}$. If $W \in W$, then $W \in \tau^{\prime}$ and int ${ }_{X} \mathrm{cl}_{x} W \supseteq$ int $_{X} \mathrm{cl}_{X} U$ for some $U \in O_{Y}^{p}$. Therefore, int ${ }_{x} \mathrm{cl}_{x} W \in O_{Y}^{p}$, whence $W \in \mathcal{U}^{p}$. Thus, $W \subseteq \mathcal{U}^{p}$. To prove the reverse inequality, let $W \in \mathcal{U}^{p}$. Then int ${ }_{X} \mathrm{cl}_{X} W \in O_{Y}^{p}$. Since int ${ }_{X} \mathrm{cl}_{X} W \supseteq$ int $_{X} \mathrm{cl}_{X}\left(\right.$ int $\left._{X} \mathrm{cl}_{X} W\right)$ it follows that int ${ }_{X} \mathrm{cl}_{X} W \in \mathcal{L}^{p}$. Hence $W \in W$. This proves the first equality in (b). The second equality follows from [9], completing the proof of the lemma.

REMARK 2.1. Since $O_{Y}^{p}=O_{Y}^{p} \#=O_{Y}^{p}+[9,10,11]$, it follows that each one of $Y, Y^{+}$and $Y^{\#}$ yield the same $\mathcal{L}^{p}$ (resp., $U^{p}$ ) for all $p \in Y$. Moreover, if $Z \in E(X)$ has the same underlying set as $Y$, and is such that $Y^{*} \leq Z \leq Y^{+}$, then $Y$ and $Z$ induce the same $\mathcal{L}^{p}$ (resp., $U^{p}$ ) for all $p \in Y$. Also, if $p \neq q$ are distinct elements of $Y$ then $\mathscr{L}^{p} \neq \mathscr{L}^{q}$ and $\mathcal{U}^{p} \neq \mathcal{U}^{q}$. Obviously, if $U \in O_{Y}^{p}$, then int ${ }_{X} \mathrm{cl}_{X}(U) \in \mathscr{L}^{p}$. Moreover, $U \in \mathcal{U}^{p}$ if and only if int ${ }_{X} \mathrm{cl}_{X}(U) \in \mathcal{U}^{p}$.

DEFINITION 2.2. Let $(Y, \tau)$ be an extension of $\left(X, \tau^{\prime}\right)$. For $G \in \tau^{\prime}$, define

$$
o_{l}(G)=G \cup\left\{p: p \in Y \backslash X, G \in \mathcal{L}^{p}\right\}
$$




$$
\begin{gathered}
o_{u}(G)=G \cup\left\{p: p \in Y \backslash X, G \in \mathcal{U}^{p}\right\} \\
a_{l}(G)=\left\{p \in Y: G \in \mathcal{L}^{p}\right\} \\
a_{u}(G)=\left\{p \in Y: G \in \mathcal{U}^{p}\right\}
\end{gathered}
$$

The proof of the Propositions 2.1, and 2.2 is straightforward.

PROPOSITION 2.1. Let $(Y, \tau)$ be an extension of $\left(X, \tau^{\prime}\right)$. Then for all $U, V \in \tau^{\prime}$

(a) $o_{l}(\varnothing)=\varnothing, o_{l}(X)=Y$,

(b) $o_{l}(U) \cap X=U$,

(c) $o_{l}(U \cap V)=o_{l}(U) \cap o_{l}(V)$,

(d) The family $\left\{o_{l}(G): G \in \tau^{\prime}\right\}$ is an open base for a Hausdorff topology $\tau_{l}$ on $Y$ and $\left(Y, \tau_{l}\right)$ is an extension of $X$.

PROPOSITION 2.2. Let $(Y, \tau)$ be an extension of $\left(X, \tau^{\prime}\right)$. Then for all $U, V \in \tau^{\prime}$,

(a) $o_{u}(\varnothing)=\varnothing$ and $o_{u}(X)=Y$,

(b) $o_{u}(U) \cap X=U$,

(c) $o_{u}(U \cap V)=o_{u}(U) \cap o_{u}(V)$,

(d) The family $\left\{o_{u}(G): G \in \tau^{\prime}\right\}$ is an open base for a Hausdorff topology $\tau_{u}$ on $Y$ and $\left(Y, \tau_{u}\right)$ is an extension of $X$.

PROPOSITION 2.3. Let $(Y, \tau)$ be an extension of $\left(X, \tau^{\prime}\right)$. Then for all $U, V \in \tau^{\prime}$

(a) $a_{l}(\varnothing)=\varnothing, a_{l}(X)=Y$,

(b) $a_{l}(U) \cap X \subseteq U$,

(c) $a_{l}(U \cap V)=a_{l}(U) \cap a_{l}(V)$,

(d) $a_{l}(U)=\cup\left\{W: W \in \tau\right.$ and $\left.\operatorname{int}_{X} \mathrm{cl}_{x}(W \cap X) \subseteq U\right\}$

(e) The family $\left\{a_{l}(G): G \in \tau^{\prime}\right\}$ is an open base for a coarser Hausdorff topology $\tau_{a l}$ on $Y, X$ is dense in $\left(Y, \tau_{a l}\right)$, but $\left(Y, \tau_{a l}\right)$ may not be an extension of $X$.

PROOF. We prove (d). The rest is straightforward. Let $p \in a_{l}(U)$. Then $U \in \mathcal{L}^{p}$. Therefore, $U \supseteq$ int $_{X} \mathrm{cl}_{X} V$ for some $V \in O_{Y}^{p}$. Therefore, there exists $W \in \tau$ such that $p \in W$ and $W \cap X=V$. It follows that int ${ }_{X} \operatorname{cl}_{X}(W \cap X) \subseteq U$. Conversely, if $W \in \tau$ is such that int ${ }_{X} \operatorname{cl}_{x}(W \cap X) \subseteq U$ and $p \in W$, then $W \cap X \in O_{Y}^{p}$. So, int ${ }_{X} \mathrm{cl}_{X}(W \cap X) \in \mathcal{L}^{p}$. This implies that $U \in \mathcal{L}^{p}$ and hence $p \in a_{l}(U)$. The proof of the proposition is now complete.

PROPOSITION 2.4. Let $(Y, \tau)$ be an extension of $\left(X, \tau^{\prime}\right)$. Then for all $U, V \in \tau^{\prime}$, (a) $a_{u}(\varnothing)=\varnothing$ and $a_{u}(X)=Y$, 
(b) $a_{u}(U) \cap X=\operatorname{int}_{X} \mathrm{cl}_{X}(U)$,

(c) $a_{u}(U \cap V)=a_{u}(U) \cap a_{u}(V)$,

(d) $a_{u}(U)=\cup\left\{W: W \in \tau\right.$ and $\left.W \cap X \subseteq \operatorname{int}_{X} \operatorname{cl}_{X}(U)\right\}$

(e) The family $\left\{a_{u}(G): G \in \tau^{\prime}\right\}$ is an open base for a coarser Hausdorff topology $\tau_{a u}$ on $Y, X$ is dense in $\left(Y, \tau_{a u}\right)$, but $\left(Y, \tau_{a u}\right)$ may not be an extension of $X$.

PROOF. We prove (d). The rest is straightforward. Let $p \in a_{u}(U)$. Then $U \in \mathcal{U}^{p}$. Therefore, int ${ }_{X} \mathrm{cl}_{X} U \in O_{Y}^{p}$. It follows that there exists $W \in \tau$ such that $p \in W$ and $W \cap X \subseteq \operatorname{int}_{X} \mathrm{cl}_{X} U$. Conversely, if $W \in \tau$ is such that $W \cap X \subseteq$ int $_{X} \mathrm{cl}_{X} U$ and $p \in W$, then $W \cap X \in O_{Y}^{p}$. So, int ${ }_{X} \mathrm{cl}_{X} U \in O_{Y}^{p}$. Therefore, $U \in \mathcal{U}^{p}$ and $p \in a_{u}(U)$.

DEFINITION 2.3. The spaces $\left(Y, \tau_{l}\right),\left(Y, \tau_{u}\right),\left(Y, \tau_{a l}\right)$, and $\left(Y, \tau_{a u}\right)$ described in propositions 2.12.4 will, henceforth, be denoted by $Y^{l}, Y^{u}, Y^{a l}$, and $Y^{a u}$ respectively. If $A \subseteq Y$, then $\operatorname{int}_{\gamma^{\prime}}(A)$ (resp. $\mathrm{cl}_{\mathrm{Y}^{\prime}}(A)$ ) will be denoted by $\operatorname{int}_{l}(A)$ (resp., $\mathrm{cl}_{l}(A)$ ). Likewise, $\operatorname{int}_{u}(A), \mathrm{cl}_{u}(A), \operatorname{int}_{a l}(A), \mathrm{cl}_{a l}(A)$, int ${ }_{a u}(A)$, and $\mathrm{cl}_{a u}(A)$ are defined in an analogous manner.

LEMMA 2.2. If $U \in \tau^{\prime}$, then

(a) $a_{l}(U) \subseteq o_{l}(U) \subseteq o_{Y}(U) \subseteq o_{u}(U) \subseteq o_{u}\left(\operatorname{int}_{X} \mathrm{cl}_{X} U\right)=a_{u}(U)=a_{u}\left(\right.$ int $\left._{X} \mathrm{cl}_{X} U\right)$,

(b) $a_{l}(U) \backslash X=o_{l}(U) \backslash X$, and $a_{u}(U) \backslash X=o_{u}(U) \backslash X$

(c) $o_{l}\left(\right.$ int $\left._{X} \mathrm{cl}_{X} U\right) \backslash X=o_{u}(U) \backslash X$, and

(d) if $U$ is regular open (i.e. $U=\operatorname{int}_{X} \mathrm{cl}_{X} U$ ), then $a_{u}(U)=a_{l}(U)$, and the equality holds in (a).

PROOF. Part (a): We show that $o_{u}\left(\right.$ int $\left._{X} \mathrm{cl}_{X} U\right)=a_{u}(U)$, the rest being straightforward. Certainly, $o_{u}\left(\right.$ int $\left._{X} \operatorname{cl}_{X} U\right) \cap X=\operatorname{int}_{X} \operatorname{cl}_{X} U=a_{u}(U) \cap X$. Let $p \in o_{u}\left(\right.$ int $\left._{X} \mathrm{cl}_{X} U\right) \backslash X$. Then int ${ }_{X} \operatorname{cl}_{X} U \in U^{p}$. Therefore, $U \in \mathcal{U}^{p}$, and $p \in a_{u}(U) \backslash X$. Conversely, let $p \in a_{u}(U) \backslash X$. Then, $U \in \mathcal{U}^{p}$. So, $p \in o_{u}(U) \backslash X \subseteq o_{u}\left(\right.$ int $\left._{X} \mathrm{cl}_{X} U\right) \backslash X$. The above arguments prove (a).

To prove (c), let $q \in o_{l}\left(\right.$ int $\left._{X} \mathrm{cl}_{X} G\right) \backslash X$. Then, int ${ }_{X} \mathrm{cl}_{X} G \in \mathscr{L}^{q}$ whence, $G \in U^{q}$. Therefore, $q \in o_{u}(G) \backslash X$. Thus, $o_{l}\left(\right.$ int $\left._{X} \operatorname{cl}_{X} G\right) \backslash X \subseteq o_{u}(G) \backslash X$. To prove the reverse inequality, let $q \in o_{u}(G) \backslash X$. Then, $G \in \mathcal{U}^{q}$, whence $\quad$ int $_{X} \mathrm{cl}_{X} G \in \mathcal{L}^{q}$. Therefore, $q \in o_{l}\left(\right.$ int $\left._{X} \operatorname{cl}_{X} G\right) \backslash X \quad$ and $o_{u}(G) \backslash X \subseteq o_{l}\left(\right.$ int $\left._{X} \mathrm{cl}_{X} G\right) \backslash X$. Hence, $o_{l}\left(\right.$ int $\left._{X} \mathrm{cl}_{X} G\right) \backslash X=o_{u}(G) \backslash X$. The rest of the lemma is straightforward.

Given a space $\left(X, \tau^{\prime}\right)$, the family $\left\{\right.$ int $\left._{X} \mathrm{cl}_{X} U: U \in \tau^{\prime}\right\}$ forms an open base for a coarser Hausdorff topology $\tau_{s}^{\prime}$ on $X$. The space $X_{s}=\left(X, \tau_{s}^{\prime}\right)$ is called the semiregularization of $X$. A space $\left(X, \tau^{\prime}\right)$ is called semiregular if $\left(X, \tau^{\prime}\right)=X_{s}$

THEOREM 2.1. If $X$ is semiregular, and $(Y, \tau)$ (not necessarily semiregular) is an extension of $X$, then $Y^{l}$ is an extension of $X$ such that $Y^{l} \leq Y$.

PROOF. If $X$ is semiregular, then $o_{l}(U)=a_{l}(U)$ for all $U \in \tau^{\prime}$. Hence, $Y^{l}$ is an extension of $X$ such that $Y^{l}=Y^{a l} \leq Y$. 
THEOREM 2.2. The spaces $Y^{a l}$ and $Y^{a u}$ are homeomorphic.

PROOF. For all $U \in \tau^{\prime}, a_{l}\left(\right.$ int $\left._{X} \mathrm{cl}_{X} U\right)=o_{u}\left(\right.$ int $\left._{X} \mathrm{cl}_{X} U\right)=a_{u}(U)$ implies that $\tau_{a u} \subseteq \tau_{a l}$. Also, if $G \in \tau^{\prime}$ and $p \in a_{l}(G)$, then $G \supseteq \operatorname{int}_{X} \operatorname{cl}_{X}(U)$ for some $U \in O_{Y}^{p} \subseteq \mathcal{U}^{p}$. Now, if $q \in a_{u}(U)$, then int $_{X} \mathrm{cl}_{X} U \in \mathcal{L}^{q}$ which implies that $G \in \mathcal{L}^{q}$, or $q \in a_{l}(G)$. Therefore, $p \in a_{u}(U) \subseteq a_{l}(G)$. Hence, $\tau_{a l} \subseteq \tau_{a u}$. This proves the theorem.

LEMMA 2.3. Let $(Y, \tau)$ be an extension of $\left(X, \tau^{\prime}\right)$. Then, for all $G \in \tau^{\prime}$ the following are true.

(a) $\mathrm{cl}_{a l}(G) \subseteq \mathrm{cl}_{l}(G)=\mathrm{cl}_{l}\left(\right.$ int $\left._{X} \mathrm{cl}_{X}(G)\right)$,

(b) $\operatorname{cl}_{u}(G)=\mathrm{cl}_{a u}(G)=\mathrm{cl}_{u}\left(\operatorname{int}_{X} \mathrm{cl}_{X} G\right)$ ),

(c) $\mathrm{cl}_{u}(G)=\mathrm{cl}_{l}(G)$,

(d) $\mathrm{cl}_{Y}(G)=\mathrm{cl}_{a u}(G)=\mathrm{cl}_{a l}\left(\right.$ int $\left._{X} \mathrm{cl}_{X}(G)\right)$, and

(e) $\mathrm{cl}_{u}\left(o_{u}(G)\right)=\mathrm{cl}_{a u}\left(a_{u}\left(\operatorname{int}_{X} \mathrm{cl}_{X}(G)\right)\right)$

PROOF. Part (a): Let $p \in \mathrm{cl}_{a l}(G)$, and let $o_{l}(U)$ be a basic open neighborhood of $p$ in $Y^{l}$. If $p \in o_{l}(U) \cap X$, then $p \in U \subseteq \operatorname{int}_{X} \mathrm{cl}_{X} U \in \mathcal{L}^{p}$. Therefore, $a_{l}\left(\right.$ int $\left._{X} \mathrm{cl}_{X} U\right)$ is an open neighborhood of $p$ in $Y^{a l}$. Consequently, $a_{l}\left(\right.$ int $\left._{X} \mathrm{cl}_{X} U\right) \cap G \neq \varnothing$. By Proposition (2.7) (b), int ${ }_{X} \mathrm{cl}_{X} U \cap G \neq \varnothing$. Hence $U \cap G \neq \varnothing$. This in turn implies that $o_{l}(U) \cap G \neq \varnothing$, and $p \in \operatorname{cl}_{l}(G)$. If $p \in O_{l}(U) \backslash X$, then $U \in \mathcal{L}^{p}$. Now, $a_{l}(U)$ is an open neighborhood of $p$ in $Y^{a l}$. Consequently, $a_{l}(U) \cap G \neq \varnothing$. Therefore, $o_{l}(U) \cap G \neq \varnothing$ whence $p \in \mathrm{cl}_{l}(G)$.

Part (b): Let $p \in \mathrm{cl}_{a u}(G)$, and let $o_{u}(U)$ be a basic open neighborhood of $p$ in $Y^{\mu}$. Since $o_{u}(U) \subseteq a_{u}(U), a_{u}(U)$ is an open neighborhood of $p$ in $Y^{a u}$. Hence, $a_{u}(U) \cap G \neq \varnothing$. Therefore, int $_{X} \mathrm{cl}_{X} U \cap G \neq \varnothing$, whence $U \cap G \neq \varnothing$. Consequently, $o_{u}(U) \cap G \neq \varnothing$. Therefore, $p \in c l_{u}(G)$. Therefore, $\operatorname{cl}_{a u}(G) \subseteq \mathrm{cl}_{u}(G)$. Conversely, let $p \in \mathrm{cl}_{u}(G)$, and let $a_{u}(U)$ be a basic open neighborhood of $p$ in $Y^{a u}$. If $p \in a_{u}(U) \cap X=\operatorname{int}_{X} \mathrm{cl}_{X} U$, then $o_{u}\left(\operatorname{int}_{X} \mathrm{cl}_{X} U\right)$ is an open neighborhood of $p$ in $Y^{u}$. Therefore, $a_{u}(U) \cap X=\operatorname{int}_{X} \operatorname{cl}_{X} U \cap G=o_{u}\left(\right.$ int $\left._{X} \mathrm{cl}_{X} U\right) \cap G \neq \varnothing$. Hence, $p \in \mathrm{cl}_{a u}(G)$. Now, if $p \in a_{u}(U) \backslash X$, then $U \in U^{p}$ and $o_{u}(U)$ is an open neighborhood of of $p$ in $Y^{\mu}$. Therefore, $o_{u}(U) \cap G \neq \varnothing$. Consequently, $a_{u}(U) \cap G \neq \varnothing$, and $p \in \mathrm{cl}_{a u}(G)$. Therefore, $\mathrm{cl}_{u}(G) \subseteq \mathrm{cl}_{a u}(G)$. Hence, $\mathrm{cl}_{u}(G) \subseteq \mathrm{cl}_{a u}(G)$. The other half of (b) is straightforward.

The proof of $(c)$ is straightforward.

Part (d): Let $p \in \mathrm{cl}_{a u}(G)$, and let $W$ be an open neighborhood of $p$ in $Y$. Then, $W \cap X \in O_{Y}^{p} \subseteq U^{p}$ shows that $o_{u}(W \cap X)$ is an open neighborhood of $p$ in $Y^{a u}$. Therefore, $a_{u}(W \cap X) \neq \varnothing$. This shows that $W \cap G \neq \varnothing$, whence $p \in \mathrm{cl}_{Y}(G)$. Conversely, let $p \in \mathrm{cl}_{Y}(G)$, and let $a_{u}(U)$ be a basic open neighborhood of $p$ in $Y^{a u}$. Then, $U \in U^{p}$. So, $o_{Y}\left(\right.$ int $\left._{X} \mathrm{cl}_{X} U\right)$ is an open neighborhood of $p$ in $Y$ such that $o_{Y}\left(\right.$ int $\left._{X} \mathrm{cl}_{X} U\right) \cap G \neq \varnothing$. This implies that $a_{u}(U) \cap G \neq \varnothing$. Hence, $p \in \mathrm{cl}_{a u}(G)$. The rest follows from (c).

THEOREM 2.3. The spaces $Y^{l} \backslash X, Y^{a l} \backslash X$, and $Y^{u} \backslash X$ are pairwise homeomorphic. 
PROOF. To prove the continuity of the identity map $i: Y^{u} \backslash X \rightarrow Y^{l} \backslash X$, let $o_{l}(G) \backslash X$ be a basic open neighborhood of $p$ in $Y^{l} \backslash X$. Then, $G \in \mathcal{L}^{p}$. Hence $G \supseteq$ int $_{X} \mathrm{cl}_{X} U$ for some $U \in O_{Y}^{p} \subseteq \mathcal{U}^{p}$. Therefore, $o_{u}(U) \backslash X$ is an open neighborhood of $p$ in $Y^{u}$ such that $o_{u}(U) \backslash X \subseteq o_{l}(G) \backslash X$. To prove that the identity map $i: Y^{l} \backslash X \rightarrow Y^{u} \backslash X$ is continuous, let $o_{u}(G) \backslash X$ be a basic open neighborhood of $p$ in $Y^{u} \backslash X$. Then $o_{l}\left(\right.$ int $\left._{X} \mathrm{cl}_{X} G\right) \backslash X$ is an open neighborhood of $p$ in $Y^{l} \backslash X$ such that $o_{l}\left(\right.$ int $\left._{X} \operatorname{cl}_{X} G\right) \backslash X=o_{u}(G) \backslash X$. Hence, the spaces $Y^{l} \backslash X$, and $Y^{u} \backslash X$ are homeomorphic. The rest of the theorem follows directly from Lemma 2.2 .

Let $Z_{1}$ and $Z_{2}$ be spaces. A map $f: Z_{1} \rightarrow Z_{2}$ is called $\theta$-continuous [3] if for every $p \in Z_{1}$ and for every open neighborhood $V$ of $f(p)$ in $Z_{2}$, there exists an open neighborhood $U$ of $p$ in $Z_{1}$ such that $f\left(\mathrm{cl}_{z_{1}} U\right) \subseteq \mathrm{cl}_{z_{2}}(V) . f$ is called perfect if $f$ is a closed map (not necessarily continuous) such that $f^{\leftarrow}(z)$ is compact in $Z_{1}$ for every $z \in Z_{2}$. Also, $f$ is called irreducible if $f$ is closed and there is no proper closed subset $K$ of $Z_{1}$ for which $f(K)=Z_{2}$. Two extensions $Z_{l}$, and $Z_{2}$ of a space $X$ are called $\theta$ - equivalent if there exists a $\theta$-homeomorphism $f$ from $Z_{I}$ onto $Z_{2}$ such that $\left.f\right|_{X}=i_{X}$, the identity map on $X$.

The next theorem depicts some of the several interrelationships between the spaces $Y, Y^{\#}, Y^{l}, Y^{\mu}$, and $Y^{a l}$.

THEOREM 2.4. Let $(Y, \tau)$ be an extension of a space $\left(X, \tau^{\prime}\right)$. The following statements are true.

(a) The identity map $i: Y^{a l} \rightarrow Y$ is perfect, irreducible and $\theta$-continuous .

(b) The identity map $i: Y^{a u} \rightarrow Y^{u}$ is perfect, irreducible and $\theta$-continuous .

(c) The identity map $i: Y^{a l} \rightarrow Y^{*}$ is $\theta$ - continuous.

(d) The identity map $i: Y^{*} \rightarrow Y^{l}$ is $\theta$-continuous.

(e) The identity map $i: Y^{*} \rightarrow Y^{u}$ is $\theta$-continuous.

(f) The identity map $i: Y^{l} \rightarrow Y^{*}$ is $\theta$-continuous .

(g) The identity map $i: Y^{u} \rightarrow Y^{\#}$ is $\theta$-continuous

(h) The identity map $i: Y^{l} \rightarrow Y^{u}$ is $\theta$-continuous .

(i) The identity map $i: Y^{u} \rightarrow Y^{l}$ is $\theta$-continuous .

(j) The identity map $i: Y^{l} \rightarrow Y$ is $\theta$-continuous .

(k) The identity map $i: Y^{u} \rightarrow Y$ is $\theta$-continuous.

(l) The identity map $i: Y^{*} \rightarrow Y^{a l}$ is $\theta$-continuous .

PROOF. Below, we outline the proofs of some parts of the theorem. The rest of the proofs are analogous.

Part (a) Since $\tau_{a l} \subseteq \tau, i: Y \rightarrow Y^{a l}$ is continuous. Hence, $i: Y \rightarrow Y^{a l}$ is irreducible and perfect. To prove the $\theta$-continuity of $i: Y^{a l} \rightarrow Y$, let $V$ be an open neighborhood of $p$ in $Y$. Then $V \cap X \in O_{Y}^{p}$ and int $_{X} \mathrm{cl}_{X}(V \cap X) \in \mathcal{L}^{p}$. Therefore, $a_{l}\left(\right.$ int $\left._{X} \mathrm{cl}_{X}(V \cap X)\right)$ is an open neighborhood of $p$ in $Y^{a l}$ such that 
$\operatorname{cl}_{a l}\left(a_{l}\left(\right.\right.$ int $\left._{X} \mathrm{cl}_{X}(V \cap X)\right)=\operatorname{cl}_{Y}\left(a_{l}\left(\operatorname{int}_{X} \operatorname{cl}_{X}(V \cap X)\right)=\operatorname{cl}_{Y}\left[a_{l}\left(\operatorname{int}_{X} \operatorname{cl}_{X}(V \cap X)\right) \cap X\right]\right.$

$=\operatorname{cl}_{Y}\left(\right.$ int $\left._{X} \operatorname{cl}_{X}(V \cap X)\right) \subseteq \operatorname{cl}_{Y}(V)$. Hence $i: Y^{a l} \rightarrow Y$ is $\theta$-continuous.

Part (b): For all $G \in \tau^{\prime}, a_{u}(G)=o_{u}\left(\right.$ int $\left._{X} \mathrm{cl}_{X} G\right) \in \tau_{u}$ shows that $i: Y^{u} \rightarrow Y^{a u}$ is continuous. Therefore, $i: Y^{a u} \rightarrow Y$ is irreducible and perfect. Let $o_{u}(G)$ be a basic open neighborhood of $p$ in $Y^{\mu}$. Since $o_{u}(G) \subseteq a_{u}(G), a_{u}(G)$ is an open neighborhood of $p$ in $Y^{a u}$ such that $\operatorname{cl}_{a u}\left(a_{u}(G)\right)=\mathrm{cl}_{u}\left(o_{u}(G)\right)$, establishing the $\theta$ - continuity of $i: Y^{a u} \rightarrow Y^{u}$.

Part (c): To prove the $\theta$-continuity of $i: Y^{a l} \rightarrow Y^{*}$, let $p \in Y$ and let $o_{Y}(G), G \in \tau^{\prime}$ be a basic open neighborhood of $p$ in $Y^{*}$. Then, $G \in O_{Y}^{p} \subseteq U^{p}$ implies that $a_{l}\left(\right.$ int $\left._{X} \mathrm{cl}_{X} G\right)$ is an open neighborhood of $p$ in $Y^{a l}$ such that $\mathrm{cl}_{a l}\left(a_{l}\left(\right.\right.$ int $\left.\left._{X} \mathrm{cl}_{X} G\right)\right) \subseteq \mathrm{cl}_{Y} \#\left(o_{Y}(G)\right)$

Part (d): Let $o_{l}(G)$ be a basic open neighborhood of $p$ in $Y^{l}$. Then, $o_{Y}(G)$ is an open neighborhood of $p$ in $Y^{\#}$ such that $\mathrm{cl}_{Y} \#\left(o_{Y}(G)\right) \subseteq \mathrm{cl}_{l}\left(o_{l}(G)\right)$, establishing the $\theta$ - continuity of $i: Y^{\#} \rightarrow Y^{l}$.

Part (h): Let $o_{u}(G)$ be a basic open neighborhood of $p$ in $Y^{\mu}$. Then, $o_{l}\left(\right.$ int $\left._{X} \mathrm{cl}_{X} G\right)$ is an open neighborhood of $p$ in $Y^{l}$ satisfying $\mathrm{cl}_{l}\left(o_{l}\left(\right.\right.$ int $\left.\left._{X} \mathrm{cl}_{X} G\right)\right) \subseteq \mathrm{cl}_{u}\left(o_{u}(G)\right.$ )

Part (1): Let $p \in Y$ and let $a_{l}(G), G \in \tau^{\prime}$ be a basic open neighborhood of $p$ in $Y^{a l}$. Then, $G \supseteq$ int $_{X} \mathrm{cl}_{X} U$ for $\quad$ some $U \in O_{Y}^{p}$. So, $\quad p \in o_{Y}(U)$. Now, $\mathrm{cl}_{Y}\left(o_{Y}(U)\right)=\mathrm{cl}_{Y}\left(o_{Y}(U) \cap X\right)=\mathrm{cl}_{Y}(U) \subseteq \mathrm{cl}_{a l}(U) \subseteq \mathrm{cl}_{a l}\left(a_{l}(G)\right)$.

We now summarize the results proved above in the following theorem.

THEOREM 2.5. The spaces $Y, Y^{*}, Y^{\prime}, Y^{\mu}$, and $Y^{a l}$, are pairwise $\theta$-homeomorphic. The spaces $Y^{\prime}$, and $Y^{\mu}$ are $\theta$-equivalent extensions of $X$ with homeomorphic remainders.

It is well known that spaces $Y$ and $Z$ are $\theta$-homeomorphic if and only if their semiregularizations are homeomorphic. [11] Hence, we have the following corollary.

COROLLARY 2.1. Let $(Y, \tau)$ be an extension of a space $(X, \tau)$. Then, the spaces $Y_{s}, Y_{s}^{\#}, Y_{s}^{l}, Y_{s}^{u}$, and $Y_{s}^{a l}$ are pairwise homeomorphic. Moreover, $Y_{s}, Y_{s}^{l}$, and $Y_{s}^{u}$ are equivalent extensions of $X_{s}$.

\section{THE EXTENSIONS $Y^{\prime *}$, AND $Y^{\mu^{*}}$.}

In this section, we define extensions $Y^{\prime^{*}}$, and $Y^{\mu^{*}}$, analogous to the simple extension $Y^{+}$of $\left(X, \tau^{\prime}\right)$ induced by an extension $(Y, \tau)$ of $X$. The spaces $Y^{*^{*}}, Y^{a l}, Y^{\mu^{*}}$, and $Y^{a u^{*}}$ all have the same underlying set as the set $Y$. An open base for the topology $\tau_{l \bullet}$ on $Y^{l^{*}}$ (respectively, $\tau_{a l \bullet}$ on $Y^{a l^{*}}$ ) is the family $\tau^{\prime} \cup\left\{G \cup\{p\}: p \in Y \backslash X, G \in \mathcal{L}^{p}\right\}$ (respectively, $\tau^{\prime} \cup\left\{G \cup\{p\}: G \in \mathcal{L}^{p}\right\}$ ). An open base for the topology $\tau_{u^{*}}$ on $Y^{\mu^{*}}$ (respectively, $\tau_{a u}$ on $Y^{a u^{*}}$ ) is the family $\tau^{\prime} \cup\left\{G \cup\{p\}: p \in Y \backslash X, G \in \mathcal{U}^{p}\right\}$ (respectively, $\left.\tau^{\prime} \cup\left\{G \cup\{p\}: G \in U^{p}\right\}\right)$. For any $A \subset Y, \mathrm{cl}_{l^{*}}(A)$ will denote the closure of $A$ in $Y^{\nu^{*}}$, with analogous notations in other cases. The proofs of the following statements are straightforward, and we omit the details. Obviously, the spaces $Y^{l^{*}} \backslash X, Y^{u^{*}} \backslash X, Y^{a *} \backslash X$, and $Y^{a *^{*}} \backslash X$ are all discrete.

THEOREM 3.1. The spaces $Y^{\prime^{*}}$, and $Y^{\mu^{*}}$ are extensions of $\left(X, \tau^{\prime}\right)$ such that $Y^{\mu^{*}} \geq Y^{+} \geq Y^{\prime^{*}}$. The set $X$ is dense in the spaces $Y^{a l^{*}}$, and $Y^{a u^{*}}$. But, $Y^{a l^{*}}$ and $Y^{a u^{*}}$ may not be extensions of $X$. 
LEMMA 3.1. For each $G \in \tau^{\prime}, \operatorname{cl}_{l^{*}}\left(o_{l}(G)\right)=\mathrm{cl}_{l}\left(o_{l}(G)\right.$, and $\mathrm{cl}_{u^{*}}\left(o_{u}(G)\right)=\mathrm{cl}_{u}\left(o_{u}(G)\right)$.

THEOREM 3.2. Each one of the identity maps $i: Y^{+} \rightarrow Y^{u^{*}}$, and $i: Y^{l^{*}} \rightarrow Y^{+}$is $\theta$-continuous.

THEOREM 3.3. The spaces $Y^{+}, Y^{r^{*}}, Y^{\mu^{*}}, Y^{a l^{*}}$, and $Y^{a u^{*}}$ are $\theta$-homeomorphic. Moreover, $Y^{+}, Y^{\gamma^{*}}$, and $Y^{\mu^{*}}$ are $\theta$ - equivalent extensions of $X$ with homeomorphic remainders.

COROLLARY 3.1. If $(Y, \tau)$ is an extension of a space $\left(X, \tau^{\prime}\right)$, then the spaces $Y_{s}^{+}, Y_{s}^{l^{*}}, Y_{s}^{u *}, Y_{s}^{a l *}$, and $Y_{s}^{a u *}$ are homeomorphic in pairs. Moreover, the spaces $Y_{s}^{+}, Y_{s}^{l^{*}}$, and $Y_{s}^{u^{* *}}$ are equivalent extensions of $X_{s}$

REMARKS 3.1. (a) If $P$ is any property of topological spaces which is preserved under $\theta$-continuous surjections, and if $(Y, \tau)$ is a P-extension of $\left(X, \tau^{\prime}\right)$, then $Y^{\prime}, Y^{\mu}, Y^{\gamma^{*}}$, and $Y^{\mu^{*}}$ are also $\mathbf{P}$ extensions of $X$.

(b) The extensions $Y^{\prime}, Y^{\mu}, Y^{Y^{*}}$, and $Y^{\mu^{*}}$ introduced above are, in general, all distinct from $Y, Y^{\#}$, and $Y^{+}$. It would be interesting to find a characterization of spaces $Y$ for which $Y^{\#}=Y^{\prime}$. A space $Z$ is called $H$ closed if it is closed in every Hausdorff space in which it is embedded [see 11 for more details]. The Katetov (respectively, Fomin) extension of a space $\left(X, \tau^{\prime}\right)$ is the space $\kappa X$ (respectively, $\sigma X$ ) whose underlying set is the set $X \cup\{p: p$ is a free open ultrafilter on $X\}$, and whose topology has for an open base the family $\tau^{\prime} \cup\{U \cup\{p\}: U \in p$, and $p \in \kappa X \backslash X\}$ (respectively, the family $\left\{o_{k X}(U): U \in \tau^{\prime}\right\}$ ). The spaces $\kappa X$, and $\sigma X$ are $H$-closed extensions of $X$ such that $(\sigma X)^{+}=\kappa X$, and $(\kappa X)^{*}=\sigma X[3,6,11]$. In general $(\sigma X)^{l} \neq \sigma X,(\kappa X)^{u} \neq \kappa X,(\sigma X)^{\mu *} \neq \sigma X$, and $(\kappa X)^{l^{*}} \neq \kappa X$. Analogous remarks apply to the Banaschewski-Fomin-Shanin extension $\mu X[13]$ of a Hausdorff space $X$

(c) A space $Z$ is called compact like, or nearly compact if every regular open cover of $Z$ is reducible to a finite subcover. A space $X$ has a compactlike extension if and only if $X_{s}$ is Tychonoff [14]. Compactlike extensions (=near compactifications) of Hausdorff almost completely regular spaces $X$ (whence, $X_{s}$ is Tychonoff) have been constructed in [2] via EF-Proximities. For a Hausdorff space $X$ whose semiregularization $X_{s}$ is Tychonoff, a maximal compactlike extension $B X$ of $X$, satisfying $(B X)_{s}=\beta X_{s}$, is constructed in [14]. If $\left(X, \tau^{\prime}\right)$ is any Hausdorff almost completely regular space, and if $(Y, \tau)$ is any near compactification of $\left(X, \tau^{\prime}\right)$, then so are $Y^{l}, Y^{\mu}, Y^{\gamma^{*}}$, and $Y^{\mu^{*}}$.

(d) A space $Z$ is called almost real compact if every open ultrafilter on $Z$ with countable closed intersection property in $Z$ converges in $Z$ [4]. A space $Z$ is almost realcompact if and only if $Z_{s}$ is almost realcompact [12]. Almost realcompactifications of a Hausdorff space have been constructed (among others) in [7], and [12]. If $\left(X, \tau^{\prime}\right)$ is any Hausdorff space, and if $(Y, \tau)$ is any almost realcompactification of $\left(X, \tau^{\prime}\right)$, then so are $Y^{\prime}, Y^{\mu}, Y^{Y^{*}}$, and $Y^{\mu^{*}}$.

(e) A Hausdorff space $Z$ is called extremally disconnected if for each open subset $U$ of $Z, c l_{z}(U)$ is open. A space $Z$ is extremally disconnected if and only if each dense subspace of $Z$ [respectively, if and only if $Z_{s}$ ] is extremally disconnected [see 11 for more details]. A Hausdorff space $Z$ is called $s$-closed if it is $H$-closed and extremally disconnected [8]. A Hausdorff space $Z$ is $s$-closed if and only if $Z_{s}$ is $s$ closed. It is shown in [8] that every extremally disconnected space $X$ admits an $s$-closed extension, viz. 
$\mathrm{k} X$; moreover, an extension $Y$ of $X$ is $s$-closed if and only if $X$ is $C^{*}$-embedded in $Y$. If $\left(X, \tau^{\prime}\right)$ is any extremally disconnected Hausdorff space, and if $(Y, \tau)$ is any $s$-closed extension of $\left(X, \tau^{\prime}\right)$, then so are $Y^{\prime}, Y^{\mu}, Y^{\gamma^{*}}$, and $Y^{\mu^{*}}$.

\section{REFERENCES}

[1] BANASCHEWSKI, B., Extensions of topological spaces. Canad. Math. Bull. 7 (1964), 1-22.

[2] CAMMAROTO, F. and NAIMPALLY S., Near Compactifications, Math. Nachr. 146 (1990), 133-136.

[3] FOMIN, S. V., Extensions of topological spaces. Ann. Math., 44 (1943), 471-480.

[4] FROLIK, Z., On almost realcompact spaces, Bull. De Lcademeie Polonaise Des Sciences Math., Astr. et Phys. LX: 49 (1961), 247-250.

[5] KATETOV, M., A note on semiregular and nearly regular spaces. Cas. Mat. Fys. 72 (1947), 9799.

[6] KATETOV, M., On H-closed extensions of a topological spaces. Cas. Mat. Fys. 72 (1947), $97-$ 99.

[7] LIU, C. T. and STRECKER, G. E., Concerning almost real compactifications, Czech. Math. J. 22 (1977), 181-190.

[8] PORTER, J. R., Hausdorff s-closed spaces, $Q \& A$ in General Topology, Vol. 4 (1986/87)

[9] PORTER, J.R. and VOTAW, C., H-closed extensions I. General Topology and Appl., 3 (1973), 211-224.

[10] PORTER, J.R. and VOTAW, C., H-closed extensions II. Trans. Amer. Math. Soc., 202 (1975), 193-209.

[11] PORTER, J. R and WOODS, R. G., Extensions and absolutes of Hausdorff spaces. SpringerVerlag, New York, 1987.

[12] TIKOO, M., Absolutes of almost realcompactifications, J. Austral. Math. Soc.(series A), 41(1986), 251-267.

[13] TIKOO, M., The Banaschewski-Fomin-Shanin Extension $\mu X$, Topology Proceedings, Vol. 10 (1985), 187-206.

[14] VERMEER, J., Minimal Hausdorff and compactlike spaces, Top. Structures II, Math. Centre Tracts 116 (1979), 271-283. 


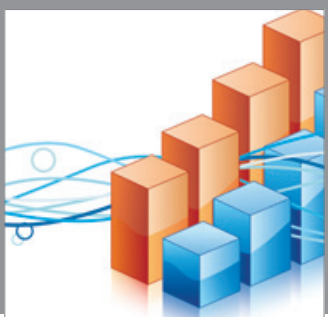

Advances in

Operations Research

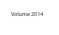

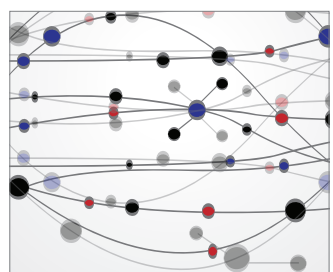

\section{The Scientific} World Journal
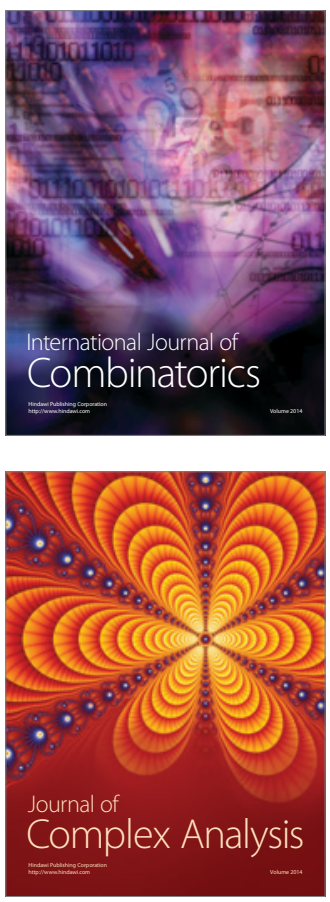

International Journal of

Mathematics and

Mathematical

Sciences
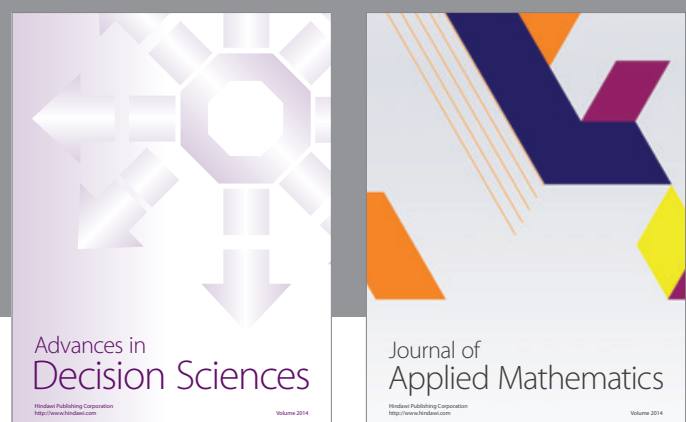

Journal of

Applied Mathematics
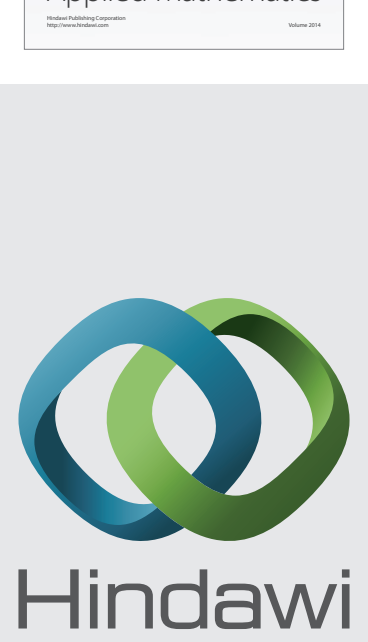

Submit your manuscripts at http://www.hindawi.com
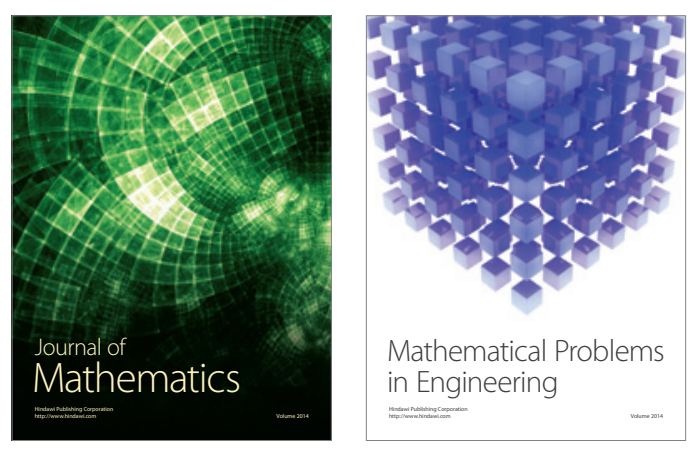

Mathematical Problems in Engineering
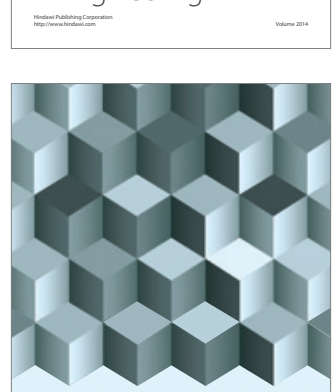

Journal of

Function Spaces
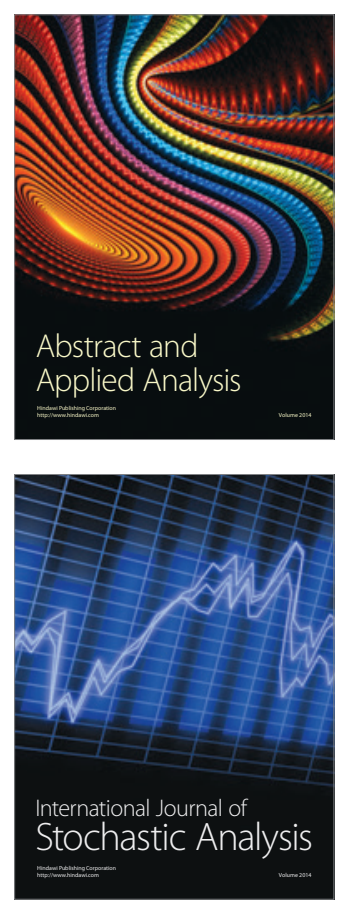

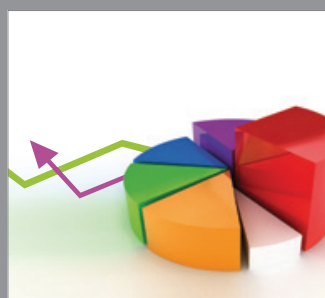

ournal of

Probability and Statistics

Promensencen
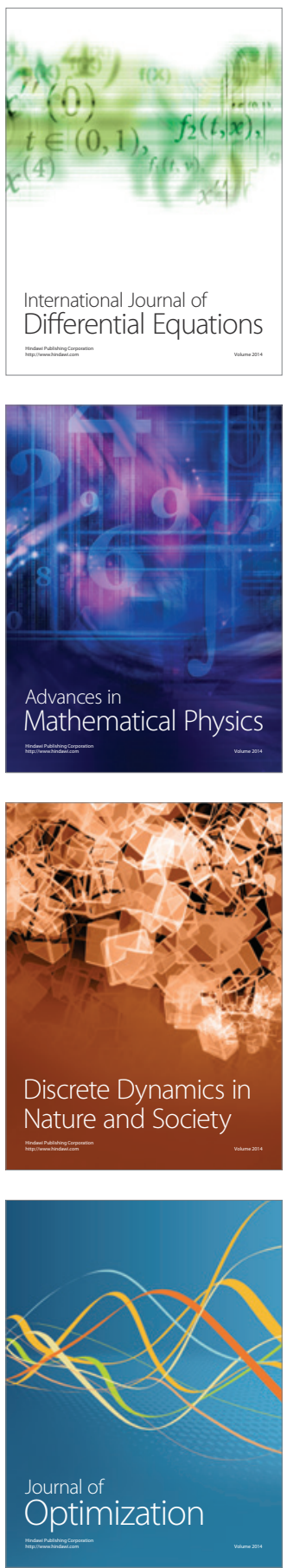